\title{
PROCESO DE ADAPTACIÓN DE UNA INTERVENCIÓN MOTIVACIONAL PARA USO DE ANTICONCEPTIVOS EN MADRES ADOLESCENTES MEXICANAS
}

\author{
PROCESS OF ADAPTING A MOTIVATIONAL INTERVENTION FOR CONTRACEPTIVE USE IN MEXICAN \\ TEENAGE MOTHERS
}

Natalia I. Manjarres-Posada, Raquel A. Benavides-Torres, Dora J. Onofre-Rodríguez, Guadalupe F. Pérez, María T. Urrutia y Rosalva del C. Barbosa-Martínez

\begin{abstract}
Objetivo. El embarazo en adolescentes es un problema en América Latina que requiere la implementación de intervenciones efectivas. Este estudio describe el proceso de adaptación de la Intervención Motivacional Asistida por Computador (CAMI) para el incremento del uso de anticonceptivos y la reducción de embarazos subsecuentes en el contexto de madres adolescentes al Norte de México. Método. Se aplicó el Modelo de Adaptación de Intervenciones de McKleroy: 1) valoración del contexto local; 2) evaluación de la intervención con expertos; 3) preparación del contenido, actividades y materiales; 4) prueba piloto de la versión adaptada; e 5) implementación, utilizando un diseño exploratorio y descriptivo. Resultados. Se obtuvo la versión CAMI-Spa con modificaciones y una tendencia favorable de las puntuaciones en variables de anticoncepción (pretest vs el postest) como actitud [120.67(DE \pm 12.36 ) vs 138.40 (DE \pm 7.20$)]$, conocimientos [28.00( $\mathrm{DE} \pm 8.76$ ) vs 44.00(DE \pm 8.49 )], procesos de cambio [78.67( $\mathrm{DE} \pm 20.83$ ) vs 108.60(DE \pm 9.21 ] y autoeficacia [43.00(DE \pm 10.37 ) vs 56.80 (DE \pm 17.38$)$ ]. Conclusiones. El uso de un modelo de adaptación de intervenciones permitió el ajuste de la intervención al contexto de las madres adolescentes mexicanas sin afectar sus componentes claves, que podría asegurar el efecto en el incremento del uso de anticonceptivos.
\end{abstract}

Palabras clave: anticoncepción, embarazo en la adolescencia, méxico, promoción de la salud, salud sexual.

Aim. Adolescent pregnancy is a problem in Latin America that requires the implementation of effective interventions. This study describes the adaptation process of Computer Assisted Motivational Intervention (CAMI) for increasing contraceptive use and reducing subsequent pregnancies in the context of adolescent mothers in Northern Mexico. Methods. The McKleroy Intervention Adaptation Model was applied: 1 ) assessment of the local context; 2) evaluation of the intervention with experts; 3 ) preparation of the content, activities and materials; 4) pilot testing of the adapted version; and 5) implementation, with an exploratory and descriptive design. Results. The CAMI-Spa version was obtained with modifications and a favorable trend in scores on contraceptive variables (pretest vs. posttest) as attitude [120.67(SD \pm 12.36 ) vs. 138.40 (SD \pm 7.20 )], knowledge [28.00(SD \pm 8.76$)$ vs $44.00(S D \pm 8.49)$ ], processes of change [78.67(SD \pm 20.83$)$ vs $108.60(S D \pm 9.21)$ ] and self-efficacy $[43.00(S D \pm 10.37)$ vs $56.80(S D \pm 17.38)]$. Conclusion. The use of an intervention adaptation model allowed for the adjustment of the intervention to the context of Mexican

Natalia Isabel Manjarres-Posada, Universidad de Guadalajara, Departamento de Salud Pública, México, orcid.org/0000-0003-2143$\underline{0897 .}$.

Raquel A. Benavides-Torres, Universidad Autónoma de Nuevo León, Facultad de Enfermería, CIDICS, México, correspondencia a rabenavi@gmail.com, raquel.benavidestr@uanl.edu.mx, orcid.org/0000-0001-5113-4250.

Dora Julia Onofre-Rodríguez, Universidad Autónoma de Nuevo León, Facultad de Enfermería, CIDICS, México, orcid.org/0000-00031214-9761.

Fabiola Pérez Baleón, Universidad Nacional Autónoma de México, Escuela de Trabajo Social, México, orcid.org/0000-0001-8419$\underline{0275}$.

María Teresa Urrutia Soto, Universidad Andrés Bello, Facultad de Enfermería, Chile, orcid.org/0000-0003-4671-1353.

Rosalva C. Barbosa-Martínez, Universidad Autónoma de Nuevo León, Facultad de Enfermería, CIDICS, México, orcid.org/0000-00034868-0722. 
Natalia I. Manjarres-Posada, Raquel A. Benavides-Torres, Dora J. Onofre-Rodríguez, Guadalupe F. Pérez, María T. Urrutia y Rosalva del C. Barbosa-Martínez

adolescent mothers without affecting its key components, which could ensure the effect on increased contraceptive use.

Keywords: contraception, health Promotion, Mexico, pregnancy in adolescence, sexual health.

El embarazo en adolescentes es un problema global de salud pública con consecuencias adversas a nivel materno, perinatal y socioeconómico. Esta situación empeora cuando la adolescente se embaraza nuevamente porque aumenta su exposición a estos riesgos (Salinas et al., 2014). Según la Organización Mundial de la salud (2018), 12.8 millones de adolescentes entre 15 y 19 años tienen un nacido vivo cada año en países de medianos y bajos ingresos. América Latina y el Caribe (ALyC) es la segunda región con mayor fecundidad adolescente en el mundo y se proyecta que continuará esta tendencia entre el 2020 y 2100 (Salinas et al. 2014).

Pese a los esfuerzos de salud pública en promoción de la anticoncepción, en algunos países de ALyC el uso de métodos anticonceptivos por mujeres adolescentes es del $43 \%$ y la mitad de quienes no los usan han experimentado un embarazo (Dongarwar \& Salihu, 2019). En México, según la Encuesta Nacional de Factores Determinantes del Embarazo Adolescente (ENFaDEA, 2017), el 78.1\% de las adolescentes no usaron un método anticonceptivo cuando se embarazaron por primera vez (Pérez et al., 2019). Esto muestra la necesidad de implementar intervenciones basadas en evidencia que han sido exitosas en otros contextos.

Las intervenciones basadas en evidencia (IBE) son estrategias que pueden implicar la manipulación de procesos cognitivos, conductuales, físicos, ambientales y / o psicosociales para mejorar los resultados de una población objetivo. Dichas intervenciones han sido probadas en investigaciones rigurosas, con fuerte validez interna, imparciales, generalizables que pueden ser aplicadas y adoptadas en diferentes individuos, poblaciones y/o contextos. La elección de una IBE requiere una revisión sistemática de literatura y la evaluación de su ajuste al fenómeno de estudio, con el propósito de definir la necesidad de adaptación, modificación o reforzamiento en el nuevo contexto de aplicación (Gitlin \& Czaja, 2016).

Entre las intervenciones efectivas para prevenir el embarazo en adolescentes se encuentra la Intervención Motivacional Asistida por Computador (CAMI), una estrategia que tiene como propósito motivar el uso consistente de anticonceptivos en madres adolescentes (Barnet et al., 2009), basada en la Entrevista Motivacional (EM), un estilo terapéutico centrado en la persona que aborda la ambivalencia ante el cambio (Miller \& Rollnick, 2015) y el Modelo Transteórico de Cambio de Conducta (Prochaska \& Prochaska, 2019) para evaluar la disposición el cambio hacia el uso de anticonceptivos mediante su constructo de etapas de cambio. El CAMI ha demostrado una alta calidad metodológica, con resultados significativos en la reducción de embarazos subsecuentes y ser costo-efectiva en comparación con otros programas similares (Barnet et al., 2010). Esta intervención se ha replicado en mujeres y hombres jovenes estadounidenses, del área urbana y en idioma inglés (Bell et al., 2018; Gold et al., 2016). Sin embargo, no existen reportes de su replicación en el contexto latinoamericano.

La implementación de una IBE de un contexto a otro requiere adaptarla a sus normas sociales y culturales para la optimización de los resultados (Norton et al., 2017). La adaptación de una IBE es un proceso activo mediante el cual esta innovación es modificada o adecuada culturalmente, sin afectar sus componentes centrales, para su adopción e implementación en un contexto diferente al original (McKleroy et al., 2006). Existen diferentes marcos teóricos que fundamentan este proceso, este estudio se basó en el Modelo de Adaptación de Intervenciones, uno de los marcos teórico-conceptuales más utilizados, para ajustar el CAMI a los valores, 
creencias y necesidades de las madres adolescentes mexicanas y lograr una mayor adherencia de la intervención durante su implementación (Lane-Fall, Curran, \& Beidas, 2019; McKleroy et al., 2006). Por tanto, el objetivo de este estudio fue describir la adaptación del CAMI mediante el Modelo de Adaptación de Intervenciones (McKleroy et al., 2006) para contribuir a la adaptación de intervenciones basadas en evidencia al contexto mexicano y puede servir de referente para otros países con el mismo idioma y afinidades culturales en la meta de incrementar el uso de anticonceptivos en adolescentes.

\section{Método}

El proceso de adaptación de la versión original del CAMI se realizó entre enero y agosto de 2019 utilizando un diseño exploratorio y descriptivo. La adaptación se realizó en el contexto sociocultural del área metropolitana de Monterrey compuesta por 11 municipios del estado de Nuevo León al Norte de México, en una red de instituciones de salud que brinda atención a madres adolescentes menores de 18 años, de bajos ingresos, nivel en el cual se concentra el 57.8\% de los embarazos en la adolescencia en México (Pérez \& Lugo, 2019). El estudio fue revisado y aprobado por el Comité de Ética en Investigación de la Universidad Autónoma de Nuevo León y considera el Reglamento de la Ley General en Materia de Investigación para la Salud en México (Gobierno de la República, 1983).

\section{Modelo de Adaptación de Intervenciones}

Es un modelo teórico-conceptual que incluye cinco pasos. El primer paso consiste en la valoración de la población objetivo, sus normas sociales, creencias y tendencias recientes, e intervención considerada para la implementación, para ello, se utilizan métodos cualitativos; el segundo paso es la evaluación de la intervención, este determina si se realiza la adaptación de los componentes de la intervención, su adopción sin adaptación o si se elige otra intervención mediante la revisión con expertos; el tercer paso es la preparación de la intervención e incluye la adaptación y prueba de los materiales de la intervención y el trabajo colaborativo con las personas interesadas; el cuarto paso consiste en realizar la prueba piloto de la intervención adaptada y el desarrollo del plan de implementación con un grupo de madres adolescentes. Por último, el quinto paso de implementación consiste en llevar a cabo la intervención con revisiones menores. Cada uno de los pasos integra circuitos de retroalimentación, procesos de monitorización, evaluación y supervisión para asegurar la orientación adecuada.

\section{Participantes}

Para cada fase se seleccionaron diferentes participantes a través de un muestreo por conveniencia. En la fase de valoración se invitaron madres adolescentes $(n=10)$, acorde con el rango propuesto por Creswell (2014), menores de 18 años, en periodo postparto inferior a un año y se excluyeron a quienes manifestaron haber presentado muerte perinatal o neonatal; en la evaluación de la intervención se consideraron expertos $(n=6)$ en embarazo en la adolescencia, EM e intervenciones conductuales; en la preparación de los materiales se consideró la participación de jóvenes universitarios $(n=5)$ entre 18 y 25 años de edad; y en la prueba piloto se incluyeron madres adolescentes $(n=6)$, según McKleroy et al. (2006) ésta se puede implementar con menos de 10 participantes.

Intervención Motivacional Asistida por Computador (CAMI) 
Es una intervención basada en el Modelo Transteórico (Prochaska \& DiClemente, 2005) y en el enfoque de EM (Miller \& Rollnick, 2015) que promueve el uso consistente de anticonceptivos en madres adolescentes para reducir el embarazo adolescente subsecuente (Barnet et al., 2009). Se obtuvo la licencia de traducción de la intervención y se realizó traducción del manual de intervención del inglés al español por una profesional de la salud bilingüe con revisión por otro profesional para detectar discrepancias entre la versión original y traducida. El CAMI incluye tres componentes: a) la evaluación de riesgo mediante un programa por computador, que contiene preguntas de valoración del riesgo de embarazo, Infecciones de Transmisión Sexual (ITS) y las etapas de cambio; b) la EM que integra discusiones sobre metas a futuro, experiencias sexuales, uso de métodos anticonceptivos y prevención de embarazo e ITS; y c) la creación de un plan seguro.

Estos componentes son implementados a través de 13 pasos en interacción con las madres adolescentes que incluyen el llenado de la evaluación de riesgo, revisión de los resultados, discusión de las metas a futuro, revisión de las experiencias sexuales y uso de anticonceptivos pasado y actual, síntesis de la discusión sobre el uso de métodos anticonceptivos, revisión y síntesis de la evaluación de riesgo de embarazo e infecciones de transmisión sexual, disposición para el uso de anticonceptivos, pros y contras del uso de anticonceptivos, discusión del nivel de importancia y confianza en la prevención de embarazo e infecciones de transmisión sexual y síntesis y creación del plan seguro para la participante. La intervención consta de ocho sesiones de 60 minutos cada una, con un intervalo de dos meses, cara a cara, para entregarse a adolescentes de 12 a 18 años, embarazadas o en postparto en visita domiciliaria. Los materiales didácticos contienen un programa por computador, guía del usuario, manual del consejero y hojas de trabajo.

\section{Procedimiento}

El reclutamiento se realizó en dos escenarios diferentes. En el primero, se identificaron madres adolescentes en una clínica de primer nivel, que cumplieran con los criterios en su expediente clínico y se invitaron mediante llamada telefónica, visita domiciliaria y publicación de carteles; en el segundo, se revisó el censo diario de pacientes en un hospital de tercer nivel, la investigadora principal y una asistente visitaron a las participantes potenciales en su unidad. Se firmó el asentimiento y consentimiento informado, se acordó previamente la fecha, lugar y hora de la participación y se ofreció una tarjeta de regalo ( US\$ 8).

Las participantes para el proceso de adaptación fueron reclutadas a través del censo de mujeres embarazadas menores de 20 años $(n=165)$ de las cuales se seleccionaron 90 registros. Posterior a la verificación de criterios se contactaron 18 participantes potenciales por teléfono y visita domiciliaria. La tasa de reclutamiento por vía telefónica fue del 8.3\%, en la mayoría de los casos las llamadas no fueron respondidas; del $22 \%$ para las visitas domiciliarias, debido a que la dirección no se encontró o no vivía allí y en la publicación de carteles no hubo respuesta. En el hospital de tercer nivel, se hizo mediante el listado diario de pacientes, se visitaron 25 pacientes identificadas y se les proporcionó información acerca del estudio. Se obtuvo una tasa de reclutamiento del 35\%, las participantes refirieron no estar interesadas, presentar síntomas desagradables postparto, o no se obtuvo el consentimiento del tutor.

En el paso de valoración se realizaron entrevistas semiestructuradas a madres adolescentes, cara a cara, mediante un guion temático diseñado por la investigadora principal, validado por dos expertos y probado por dos pares adolescentes para conocer aspectos sociodemográficos, la 
experiencia de ser madres, el uso de métodos anticonceptivos, necesidades y perspectivas sobre su participación en un estudio de intervención. Cada entrevista duró 20 minutos, fue audiograbada, previa autorización de las participantes y se identificaron con un folio (e.g. P01) para mantener la confidencialidad. La evaluación de la intervención se realizó en sesiones con expertos mediante análisis de los resultados de la fase anterior y la congruencia con los componentes y actividades del CAMI para definir su adopción con o sin modificaciones. En la preparación de los materiales se desarrollaron actividades de diseño, revisión y actualización basados evidencia científica reciente y en sesiones grupales con estudiantes. En la prueba piloto, se realizaron dos sesiones con madres adolescentes para evaluar la adaptación cultural, del lenguaje, aceptabilidad y fidelidad (Márquez, 2012) con escalas Likert; y probar variables de interés como actitud anticonceptiva, conocimientos en anticoncepción, procesos de cambio para el uso de anticonceptivos, balance decisional para el uso de anticonceptivos, autoeficacia anticonceptiva y uso de anticonceptivos mediante la aplicación de instrumentos de medición validados en el pretest y postest. La implementación, quinto paso de la adaptación será llevado a cabo mediante un ensayo clínico aleatorizado en otro estudio.

\section{Análisis de datos}

En la fase de valoración se transcribieron las grabaciones, se procesaron en el programa MAXQDA y se realizó el análisis de contenido por dos investigadores, mediante un enfoque deductivo, con lectura a profundidad de los discursos en repetidas ocasiones, seguido de: 1) creación de códigos, definición, revisión y modificaciones, y estructuración en lista; y 2) agrupación en categorías más amplias (Miles et al., 2014). En el análisis cuantitativo sobre las características de las participantes en cada una de las fases del proceso de adaptación se usó estadística descriptiva. Los datos de la prueba piloto fueron procesados con el paquete estadístico Social Package for the Social Sciences y el análisis, utilizando estadísticos de tendencia central y de dispersión con el fin de estimar las tendencias en las variables de interés en el pretest y postest de la intervención.

\section{Resultados}

\section{Resultados cualitativos}

Las madres adolescentes tuvieron en promedio 16.7 ( $D E \pm 0.48$ ) años y nivel de escolaridad secundaria, fueron entrevistadas en periodo postparto tardío $(n=3)$ a través de visitas domiciliarias y en postparto inmediato $(n=7)$ en el ámbito hospitalario en una habitación privada disponible o en la unidad de la participante previa autorización suya.

Con relación a sus antecedentes personales y reproductivos, el $80 \%$ de las participantes tenían pareja, no había usado métodos anticonceptivos previo a su embarazo, eran primigestas y la mitad de ellas reportaron vida sexual activa. De los discursos emergieron cuatro categorías: ser madre, uso de métodos anticonceptivos, metas a futuro y atención en salud sexual y reproductiva que orientaron el proceso de adaptación cultural y del lenguaje (Tabla 1). 
Natalia I. Manjarres-Posada, Raquel A. Benavides-Torres, Dora J. Onofre-Rodríguez, Guadalupe F. Pérez, María T. Urrutia y Rosalva del C. Barbosa-Martínez

Tabla 1

Análisis de contenido de entrevistas semiestructuradas y aportaciones

\begin{tabular}{|c|c|c|c|}
\hline Categorías & Códigos & Discursos ${ }^{a}$ & Aportaciones a la adaptación \\
\hline Ser madre & $\begin{array}{l}\text { Impacto } \\
\text { Cambios en la } \\
\text { vida } \\
\text { Sentimientos } \\
\text { hacia su hijo }\end{array}$ & $\begin{array}{l}\text { “...me afectó en todo por ser } \\
\text { menor de edad, me faltaban } \\
\text { más cosas por hacer, estudiar y } \\
\text { disfrutar... ahora me dedico a la } \\
\text { bebé, a limpiar, a recoger, a } \\
\text { atenderla" (P02). }\end{array}$ & $\begin{array}{l}\text { Identificación de motivadores } \\
\text { para la prevención de } \\
\text { embarazo. } \\
\text { ejemplos de dialogo en el } \\
\text { manual del facilitador. }\end{array}$ \\
\hline Uso de MAC & $\begin{array}{l}\text { Opinión sobre } \\
\text { los MAC } \\
\text { Creencias } \\
\text { sobre MAC } \\
\text { Razones para el } \\
\text { uso de MAC } \\
\text { Percepciones } \\
\text { de los otros }\end{array}$ & $\begin{array}{l}\text { "que debemos de usarlos } \\
\text { cuando ya tenemos una } \\
\text { relación sexual, más en las } \\
\text { adolescentes como yo" (P08). } \\
\text { "dicen que te lo tienes que } \\
\text { revisar [DIU] y hacer el } \\
\text { Papanicolaou [citología } \\
\text { cervical] cada mes" (P05). } \\
\text { "pienso que después no van a } \\
\text { poder tener hijos [Pastillas]" } \\
\text { (P10). } \\
\text { "para no volver a tener familia } \\
\text { sin planificarla" (P09). }\end{array}$ & $\begin{array}{l}\text { Comprensión de los } \\
\text { conocimientos previos, } \\
\text { creencias, normas sociales y } \\
\text { personas que influyen en las } \\
\text { decisiones sobre el uso de } \\
\text { MAC. } \\
\text { Adecuación de la intervención } \\
\text { a las necesidades de las } \\
\text { madres adolescentes. }\end{array}$ \\
\hline Metas a futuro & $\begin{array}{l}\text { En cinco años } \\
\text { Como lograrlo } \\
\text { Obstáculos }\end{array}$ & $\begin{array}{l}\text { “con mi hija, con un negocio ... } \\
\text { [Obstáculos] pues en parte la } \\
\text { economía" (P08). }\end{array}$ & $\begin{array}{l}\text { Contextualización del plan de } \\
\text { vida } \\
\text { Inclusión de suplementos } \\
\text { sobre formación académica y } \\
\text { becas }\end{array}$ \\
\hline $\begin{array}{l}\text { Atención en } \\
\text { salud sexual y } \\
\text { reproductiva }\end{array}$ & $\begin{array}{l}\text { Memorias de la } \\
\text { información } \\
\text { Satisfacción en } \\
\text { la atención }\end{array}$ & $\begin{array}{l}\text { “...me dijeron que el DIU era } \\
\text { muy bueno, que aceptara, } \\
\text { porque yo me iba a poner el } \\
\text { implante" (P05). } \\
\text { "pues no me gustó nada, } \\
\text { porque yo quería que me } \\
\text { explicaran más y yo le decía a la } \\
\text { enfermera, y nunca fue para } \\
\text { explicarme" (P05). }\end{array}$ & $\begin{array}{l}\text { Evaluación de la congruencia } \\
\text { de la intervención con las } \\
\text { expectativas de las MA } \\
\text { Diseño de fichas informativas } \\
\text { sobre MAC e ITS. } \\
\text { Reforzamiento del uso de } \\
\text { lenguaje claro y sencillo. }\end{array}$ \\
\hline
\end{tabular}

Nota. ${ }^{a}$ Segmentos de los discursos obtenidos en las entrevistas

Con respecto a sus perspectivas de participación, las participantes expresaron la necesidad de recibir información sobre los métodos anticonceptivos, su uso, demostraciones y fallos; y orientación sobre la elección del método anticonceptivo más apropiado a su caso. También expresaron interés en aprender cómo diseñar un plan de vida, planificar una familia y sobre ITS. Con respecto a la entrega de la intervención, las participantes reportaron que serían adecuadas 2.9 sesiones, de 56.8 minutos cada una, 60\% prefirieron el centro de salud como lugar para la implementación y 40\% que fueran grupales. El 60\% prefirieron los cuestionarios en formato de lápiz y papel y sugirieron una canasta de regalos para el bebé como compensación. 
Los expertos de la ENFaDEA 2017 expresaron la pertinencia del CAMI para la atención en salud de las adolescentes, recomendaron explorar fallos y creencias sobre los métodos anticonceptivos y la posibilidad de integrar a la pareja en la intervención. El experto en Entrevista Motivacional valoró la traducción y vigencia de este componente y el manual del consejero, recomendó su actualización e inclusión de nuevos conceptos y brindó entrenamiento y práctica en Entrevista Motivacional y, por último, los expertos en intervenciones conductuales sugirieron el abordaje de normas subjetivas. Como resultado del proceso de adaptación se adoptó el CAMI con modificaciones (Tabla 2). A partir de esta fase se denominó CAMI-Spa por su versión en español.

Tabla 2

Matriz de adaptaciones al CAMI

\begin{tabular}{|c|c|c|c|c|}
\hline Componente & $\begin{array}{l}\text { Actividad/ } \\
\text { Contenido }^{a}\end{array}$ & Versión original & $\begin{array}{l}\text { Descripción de la } \\
\text { adaptación }\end{array}$ & Justificación b \\
\hline $\begin{array}{l}\text { Programa por } \\
\text { computador } \\
\text { CAMI }\end{array}$ & $\begin{array}{l}\text { Resumen de } \\
\text { la evaluación } \\
\text { de riesgo } \\
\text { (Paso 2) }\end{array}$ & $\begin{array}{l}\text { Impresión del } \\
\text { resumen de la } \\
\text { evaluación de } \\
\text { riesgo }\end{array}$ & $\begin{array}{l}\text { Generación del } \\
\text { resumen en } \\
\text { formato } \\
\text { electrónico }\end{array}$ & $\begin{array}{l}\text { El uso del resumen } \\
\text { electrónico disminuye el } \\
\text { requerimiento de equipos } \\
\text { durante la sesión. }\end{array}$ \\
\hline \multirow[t]{2}{*}{$\begin{array}{l}\text { EM por } \\
\text { etapas }\end{array}$} & $\begin{array}{l}\text { Revisión del } \\
\text { historial de } \\
\text { uso de MAC } \\
\text { (Paso 6) }\end{array}$ & $\begin{array}{l}\text { Sin descripción } \\
\text { de material } \\
\text { didáctico de } \\
\text { apoyo }\end{array}$ & $\begin{array}{l}\text { Diseño e inclusión } \\
\text { de mapa temático, } \\
\text { fichas informativas } \\
\text { y muestrario sobre } \\
\text { MAC }\end{array}$ & $\begin{array}{l}\text { Una guía visual facilita la } \\
\text { identificación de } \\
\text { experiencias pasadas, la } \\
\text { discusión sobre MAC, y la } \\
\text { entrega de la información } \\
\text { por los facilitadores. }\end{array}$ \\
\hline & $\begin{array}{l}\text { Revisión de } \\
\text { los resultados } \\
\text { de la } \\
\text { evaluación de } \\
\text { riesgo de } \\
\text { embarazo e } \\
\text { ITS (Paso 8) }\end{array}$ & $\begin{array}{l}\text { Sin descripción } \\
\text { de material } \\
\text { didáctico de } \\
\text { apoyo }\end{array}$ & $\begin{array}{l}\text { Diseño de mapa } \\
\text { temático y fichas } \\
\text { informativas sobre } \\
\text { ITS }\end{array}$ & \multirow{6}{*}{$\begin{array}{l}\text { Miller \& Rollnick (2015) } \\
\text { incluyen nuevo } \\
\text { conocimiento sobre el } \\
\text { espíritu, procesos y } \\
\text { resultados de la EM, } \\
\text { neurolingüística del cambio } \\
\text { y como los profesionales } \\
\text { aprenden aplicar la EM. }\end{array}$} \\
\hline \multirow{5}{*}{$\begin{array}{l}\text { Manual del } \\
\text { consejero }\end{array}$} & \multirow{5}{*}{$\begin{array}{l}\text { Descripción } \\
\text { general de la } \\
\text { EM }\end{array}$} & \multirow{5}{*}{$\begin{array}{l}\text { Cinco principios } \\
\text { claves de la EM } \\
\text { Técnicas } \\
\text { motivacionales } \\
\text { Habilidades } \\
\text { básicas de la EM }\end{array}$} & $\begin{array}{l}\text { Actualización } \\
\text { como: }\end{array}$ & \\
\hline & & & Inclusión del & \\
\hline & & & compasión & \\
\hline & & & Método de la EM & \\
\hline & & & $\begin{array}{l}\text { Microhabilidades } \\
\text { de la EM }\end{array}$ & \\
\hline $\begin{array}{l}\text { Entrega de la } \\
\text { intervención }\end{array}$ & $\begin{array}{l}\text { Número de } \\
\text { sesiones }\end{array}$ & Ocho sesiones & Dos sesiones & $\begin{array}{l}\text { El CAMI original sugiere que } \\
\text { dos sesiones pueden } \\
\text { reducir el embarazo } \\
\text { subsecuente(Barnet et al., } \\
\text { 2009). Una sesión sobre uso } \\
\text { del condón tiene el } \\
\text { potencial para promover el } \\
\text { cambio de conducta en los }\end{array}$ \\
\hline
\end{tabular}


Natalia I. Manjarres-Posada, Raquel A. Benavides-Torres, Dora J. Onofre-Rodríguez, Guadalupe F. Pérez, María T. Urrutia y Rosalva del C. Barbosa-Martínez

\begin{tabular}{|c|c|c|c|c|}
\hline Componente & $\begin{array}{l}\text { Actividad/ } \\
\text { Contenido }^{a}\end{array}$ & Versión original & $\begin{array}{l}\text { Descripción de la } \\
\text { adaptación }\end{array}$ & Justificación b \\
\hline & & & & $\begin{array}{l}\text { jóvenes (Whitaker et al., } \\
\text { 2016). }\end{array}$ \\
\hline
\end{tabular}

Nota. ${ }^{\text {a }}$ Tomado del manual de consejero CAMI.

${ }^{\mathrm{b}}$ Extraída de expertos y evidencia científica

Preparación de la adaptación

El material didáctico fue diseñado por estudiantes mexicanos, con una edad promedio de 22.8 (DE \pm 0.48 ) años, incluyó mapas temáticos sobre métodos anticonceptivos e ITS, fichas con la definición, mecanismo de acción, eficacia, modo de uso, ventajas y desventajas, fallos y creencias de los métodos anticonceptivos; las fichas de ITS incluyeron definición, signos y síntomas, tratamiento y prevención y el manual del consejero fue actualizado por la investigadora principal.

\section{Resultados cuantitativos}

Prueba piloto

Se realizó con madres adolescentes $(n=6)$ que reportaron tener en promedio 16.8 años de edad ( $D E \pm 0.40$ ), 97.3 días ( $D E \pm 19.5$ ) de periodo postparto y 9 años ( $D E \pm 1.0$ ) de estudio en la primera sesión; el $66.7 \%$ indicó estar en unión libre con el padre de su hijo y el $83.3 \%$ dedicarse actividades en el espacio doméstico. En sus antecedentes reproductivos informaron ser primigestas, el 50\% tener vida sexual activa y el 33.3\% haber usado un método anticonceptivo en el pasado.

La primera sesión del CAMI-Spa se realizó después de la aplicación del pretest y se solicitó a las participantes su opinión y recomendaciones en tres momentos diferentes de la sesión. La segunda sesión se realizó con un tiempo promedio de 15.7 días ( $D E \pm 3.0$ ) y se agregó una tercera sesión de 38.8 días ( $D E \pm 8.0$ ) con respecto a la primera sesión dado que no se observaron cambios en la variable de conducta anticonceptiva, posiblemente por el corto tiempo entre la primera y segunda sesión, la presencia del ciclo menstrual para el inicio o cambio de un método anticonceptivo y/o el acceso a los servicios de salud.

Cuatro madres adolescentes refirieron que dos sesiones eran adecuadas y en dos casos que podrían ser tres a cinco sesiones. Con respecto al material didáctico recomendaron que las fichas de los métodos anticonceptivos fuesen a color y el consejero sugirió la modificación de palabras como (in)fertilidad y prevención, mayor síntesis de la información e inclusión de frases sobre derechos sexuales y reproductivos. Las participantes calificaron favorablemente el CAMI-Spa indicando que el lenguaje era claro, coherente con el tema y no ofensivo; con respecto a su satisfacción, el 100\% de las madres adolescentes reportaron que les gustó el programa, les pareció útil, se sintieron satisfechas, respondió a sus expectativas y lo recomendarían amigos y parientes.

Por otra parte, se evaluó comparativamente la tendencia de las variables de interés entre el pretest y postest de la segunda y tercera sesión del CAMI-Spa. En el postest de la segunda sesión se observaron cambios favorables en las variables de anticoncepción como actitud, conocimientos, procesos de cambio, en las ventajas del balance decisional y la autoeficacia, sin 
embargo, no se obtuvo en el uso de métodos anticonceptivos, esto podría relacionarse con el corto tiempo de reflexión de las participantes entre sesiones, una condición determinante en el proceso de toma de decisiones (American Counseling Association, 2015). Posterior a la tercera sesión se mantuvo la tendencia de cambio de las variables iniciales con respecto a la segunda sesión, y se incrementaron las ventajas sobre las desventajas en el balance decisional para el uso de anticonceptivos y el uso de métodos anticonceptivos (Tabla 3).

Tabla 3

Media de puntuaciones de las variables de anticoncepción entre el pretest y postest

\begin{tabular}{|c|c|c|c|c|c|c|c|c|c|c|c|c|c|c|c|}
\hline & \multicolumn{5}{|c|}{ Pretest } & \multicolumn{5}{|c|}{$\begin{array}{c}\text { Postest } \\
\text { (2 sesión - } 15.1 \text { días) }\end{array}$} & \multicolumn{5}{|c|}{$\begin{array}{c}\text { Postest } \\
\text { (3 sesión - } 38.8 \text { días) }\end{array}$} \\
\hline & $n$ & Mín & Máx & $M$ & $\mathrm{DE}$ & $n$ & Mín & Máx & $M$ & $\mathrm{DE}$ & $n^{a}$ & Mín & Máx & $M$ & $\mathrm{DE}$ \\
\hline AcA & 6 & 108 & 141 & 120.67 & 12.36 & 6 & 89 & 147 & 129.00 & 22.23 & 5 & 127 & 145 & 138.40 & 7.20 \\
\hline CA & 6 & 12 & 36 & 28.00 & 8.76 & 6 & 32 & 56 & 42.67 & 9.35 & 5 & 36 & 56 & 44.00 & 8.49 \\
\hline PcA & 6 & 37 & 93 & 78.67 & 20.83 & 6 & 63 & 121 & 99.33 & 19.81 & 5 & 99 & 123 & 108.60 & 9.21 \\
\hline $\mathrm{BdV}$ & 6 & 31 & 75 & 61.50 & 16.66 & 6 & 34 & 75 & 63.50 & 15.88 & 5 & 58 & 75 & 69.80 & 7.19 \\
\hline $\mathrm{BdD}$ & 6 & 15 & 36 & 24.83 & 7.31 & 6 & 15 & 55 & 31.33 & 14.71 & 5 & 15 & 39 & 24.00 & 11.23 \\
\hline AuA & 6 & 33 & 59 & 43.00 & 10.37 & 6 & 31 & 70 & 52.67 & 15.68 & 5 & 29 & 75 & 56.80 & 17.38 \\
\hline UM & 6 & 0 & 21 & 9.33 & 10.54 & 6 & 0 & 21 & 6.50 & 10.11 & 5 & 0 & 26 & 13.60 & 12.58 \\
\hline
\end{tabular}

Nota: $A c a=A c t i t u d$ Anticonceptiva, $C A=$ Conocimientos en Anticoncepción, PcA=Procesos de cambio: Anticoncepción,

$\mathrm{BdV}=$ Balance decisional-Ventajas, $\mathrm{BdD}=$ Balance decisional-Desventajas, AuA=Autoeficacia Anticonceptiva, UM=Uso de MAC, Mín

= mínimo, Máx = máximo, $\mathrm{M}$ = media, $\mathrm{DE}=$ Desviación Estándar

a Una MA no aceptó recibir la tercera sesión.

\section{Discusión}

Hasta donde se tiene conocimiento, este es uno de los primeros estudios que reporta el proceso de adaptación lingüística y cultural de una intervención basada de evidencia como el CAMI para el uso de anticonceptivos en madres adolescentes mexicanas basado en el Modelo de Adaptación de Intervenciones. Como resultado se obtuvo la versión adaptada CAMI-Spa que fue evaluada favorablemente por las madres adolescentes en cuanto al uso de lenguaje claro, comprensible y apropiado y su adecuación coherente culturalmente con el uso de métodos anticonceptivos y sus necesidades.

Adicionalmente, en la adaptación del CAMI-Spa se contempló la evaluación de su contenido, pedagogía e implementación a partir de la valoración del contexto de las madres adolescentes y del entrenamiento del facilitador que condujeron a modificaciones de formato, lenguaje y adición de elementos como materiales de apoyo sobre anticoncepción e ITS en la búsqueda de una mayor sensibilidad cultural sin afectar los aspectos esenciales de la intervención. Rolleri et al. (2014) y McKleroy et al. (2006) establecen que mantener la fidelidad de los componentes claves es indispensable para conservar la efectividad de la intervención como se pretendió en esta experiencia de adaptación.

Con relación a la implementación de la intervención se presentó una discrepancia en el número de sesiones entre lo sugerido por las madres adolescentes y la intervención original para llegar a un consenso de entrega de dos sesiones que podría disminuir la efectividad de la intervención. Ortega, Giannotta, Latina, \& Ciairano (2012) también reportaron discrepancias en los tiempos de entrega en la adaptación del programa Fortalecimiento de Familias 10-14 a 
población italiana en la que priorizaron la forma como sus participantes procesaban la información y la viabilidad de su implementación. Esta similitud evidencia la importancia de considerar los diferentes factores que pueden influir en la factibilidad y aceptabilidad de una intervención adaptada, tales como las necesidades de las participantes, su contexto y los recursos logísticos, económicos y de talento humano disponibles para su implementación.

Durante el proceso de adaptación se propusieron modificaciones sobre la implementación del CAMI-Spa como la inclusión de la pareja por parte de los expertos y el tipo de sesión de individual a grupal por las madres adolescentes. La inclusión de nuevas formas de participación en esta etapa puede implicar cambios a profundidad que alterarían los componentes centrales y por tanto los efectos de la intervención (Gitlin \& Czaja, 2016); así mismo el objetivo de una intervención grupal es la resolución de problemas en el campo de lo colectivo y no en la atención particular de condiciones de cada participante (Andrade, 2011). Por tanto, dado que el propósito de la etapa de desarrollo en que se encuentra la intervención es evaluar si tiene los efectos deseados, entender el marco teórico y monitorizar la fidelidad, factibilidad y aceptabilidad en su implementación estas modificaciones no se llevaron a cabo y se sugieren considerarlas para etapas posteriores.

Las perspectivas, creencias y valores de las madres adolescentes mexicanas estuvieron enmarcadas en su experiencia de la maternidad a temprana edad y el uso de métodos anticonceptivos. Se evidenció un contexto predominante de roles de género tradicionales que han sido normalizados a través del aprendizaje social de las adolescentes y que dan significado a la maternidad como un evento deseable y de alto valor social (Jiménez-González, GranadosCosme, \& Rosales-Flores, 2017). Resultados similares se presentaron en la adaptación de una intervención de educación sexual en adolescentes colombianos escolarizados que reflejaron la influencia del machismo en la conducta sexual (Morales et al., 2019). Estas perspectivas fueron incluidas como conocimiento previo a la implementación del CAMI-Spa por facilitador para incrementar la empatía hacia las participantes, facilitar la contextualización de cada uno de los pasos de la intervención y reforzar los derechos sexuales y reproductivos.

En cuanto a la atención en salud sexual y reproductiva se observó que las madres adolescentes reportaron mayor interés en conocer detalladamente la información sobre los MAC, en un espacio confiable de interacción, libre de juicios e imposición, con un proveedor de salud que atienda sus dudas e inquietudes. Estos hallazgos coinciden con un estudio realizado por de Castro et al. (2018) con adolescentes mexicanos y refuerza la relevancia de la EM como componente clave del CAMI-Spa dado que es un enfoque terapéutico que tiene un interés genuino en la postura y sabiduría de la persona y que se basa en la colaboración, aceptación, compasión y evocación (Miller \& Rollnick, 2015).

\section{Limitaciones}

El estudio se desarrolló con una muestra pequeña de participantes en diferentes momentos del periodo postparto, residentes en el área urbana, de la región norte de México debido a las limitaciones de tiempo y recursos que podrían afectar la generalización de los resultados en cuanto a los aspectos culturales de las madres adolescentes mexicanas. Se sugiere realizar futuros estudios de adaptación con madres adolescentes de contextos rurales y otras regiones. 


\section{Conclusiones}

El uso del Modelo de Adaptación de Intervenciones facilitó un marco adecuado para la adaptación lingüística y cultural del CAMI al CAMI-Spa. Proporcionó herramientas para la implementación de intervenciones en los servicios de salud como las preferencias de las madres adolescentes sobre el contenido, entrega de la intervención, cantidad y tipo de sesiones, y los componentes que se consideran esenciales en una consejería sobre métodos anticonceptivos. Esta adaptación lingüística y cultural CAMI-Spa resulta de gran relevancia dado que es uno de los primeros reportes de adaptación de una intervención basada en evidencia para el incremento del uso de anticonceptivos en madres adolescentes en el contexto latinoamericano y específicamente mexicano.

\section{Agradecimientos}

Las autoras agradecen la financiación recibida por el Consejo Nacional de Ciencia y Tecnología (CONACyT, beca 626549), al Centro de Investigación y Desarrollo en Ciencias de la Salud con los recursos requeridos y a la Subdirección de Posgrado de la Facultad de Enfermería de la Universidad Autónoma de Nuevo León por su asesoramiento en el desarrollo de este artículo.

Las autoras declaran que no existe conflicto de interés.

\section{Referencias}

American Counseling Association. (2015). The ACA Encyclopedia of Counseling. The ACA Encyclopedia of Counseling. Wiley. Recuperado diciembre 11 de 2019. doi.org/10.1002/9781119221548

Andrade, J. A. (2011). La intervención grupal: una lectura de los conceptos de Enrique Pichón Riviere: artículo de reflexión investigativa. Revista Electrónica De Psicología Iztacala, 14(3), 194-228

Barnet, B., Liu, J., DeVoe, M., Duggan, A. K., Gold, M. A., \& Pecukonis, E. (2009). Motivational intervention to reduce rapid subsequent births to adolescent mothers: A communitybased randomized trial. Annals of Family Medicine, 7(5), 436-445. doi.org/10.1370/afm.1014

Barnet, B., Rapp, T., DeVoe, M., \& Mullins, C. D. (2010). Cost-effectiveness of a Motivational Intervention to reduce rapid repeated childbearing in high-risk adolescent mothers. Archives of Pediatrics \& Adolescent Medicine, 164(4), 370-376. doi.org/10.1001/archpediatrics.2010.16

Bell, D. L., Garbers, S., Catallozzi, M., Hum, R. S., Nechitilo, M., McKeague, I. W., ... Gold, M. A. (2018). Computer-Assisted Motivational Interviewing Intervention to facilitate teen pregnancy prevention and fitness behavior changes: A randomized trial for young men. Journal of Adolescent Health, 62(3), S72-S80. doi.org/10.1016/j.jadohealth.2017.06.015

Creswell, J. W. (2014). Research design: qualitative, quantitative, and mixed methods approaches (4th ed.). Sage Publications. 
De Castro, F., Place, J. M., Allen-Leigh, B., Barrientos-Gutierrez, T., Dues, K., Eternod, M., \& Hernández-Avila, M. (2018). Perceptions of adolescent 'simulated clients' on barriers to seeking contraceptive services in health centers and pharmacies in Mexico. Sexual \& Reproductive Healthcare, 16, 118-123. doi.org/10.1016/j.srhc.2018.03.003

Dongarwar, D., \& Salihu, H. M. (2019). Influence of sexual and reproductive health literacy on single and recurrent adolescent pregnancy in Latin America. Journal of Pediatric and Adolescent Gynecology, 0(0), 1-8. doi.org/10.1016/j.jpag.2019.06.003

Gitlin, L., \& Czaja, S. (2016). Intervention Research. Designing, evaluating, and implementing. Springer Publishing Company.

Gold, M. A., Tzilos, G. K., Stein, L. A. R., Anderson, B. J., Stein, M. D., Ryan, C. M., ... DiClemente, C. (2016). A randomized controlled trial to compare Computer-assisted Motivational Intervention with Didactic Educational Counseling to reduce unprotected sex in female adolescents. Journal of Pediatric and Adolescent Gynecology, 29(1), 26-32. doi.org/10.1016/i.jpag.2015.06.001

Gobierno de la República. (1983). Reglamento de la Ley General de Salud en Materia de Investigación para la Salud. Diario Oficial de La Federación. Recuperado de http://www.salud.gob.mx/unidades/cdi/nom/compi/rlgsmis.html

Jiménez-González, A., Granados-Cosme, J. A., \& Rosales-Flores, R. A. (2017). Embarazo en adolescentes de una comunidad rural de alta marginalidad. Un estudio mixto de caso. Salud Publica de Mexico, 59(1), 11-18. doi.org/10.21149/8410

Lane-Fall, M. B., Curran, G. M., \& Beidas, R. S. (2019). Scoping implementation science for the beginner: locating yourself on the "subway line" of translational research. BMC medical research methodology, 19(1), 133. doi.org/10.1186/s12874-019-0783-z

Márquez, M. A. (2012). Intervención sobre comunicación sexual para padres en Comunidades urbano-marginadas [tesis doctoral]. Universidad Autónoma de Nuevo León.

McKleroy, V. S., Galbraith, J. S., Cummings, B., Jones, P., Harshbarger, C., Collins, C., ... Carey, J. W. (2006). Adapting evidence-based behavioral interventions for new settings and target populations. AIDS Education and Prevention, 18(SUPPL. A), 59-73. doi.org/10.1521/aeap.2006.18.supp.59

Miles, M., Huberman, M., \& Saldaña, J. (2014). Qualitative Data Analysis (3ra ed.). SAGE Publications.

Miller, W. R., \& Rollnick, S. (2015). La entrevista motivaciona : Ayudar a las personas a cambiar (3rd ed.). Grupo Planeta.

Morales, A., Garcia-Montaño, E., Barrios-Ortega, C., Niebles-Charris, J., Garcia-Roncallo, P., Abello-Luque, D., ... \& Lightfoot, M. (2019). Adaptation of an effective school-based sexual health promotion program for youth in Colombia. Social Science \& Medicine, 222, 207-215. doi.org/10.1016/i.socscimed.2019.01.011 
Norton, M., Chandra-Mouli, V., \& Lane, C. (2017). Interventions for preventing unintended, rapid repeat pregnancy among adolescents: A review of the evidence and lessons from highquality evaluations. Global Health, Science and Practice, 5(4), 547-570. doi.org/10.9745/GHSP-D-17-00131

Organización Mundial de la Salud. (2018, Agosto 20). Adolescent birth rate. http://apps.who.int/gho/data/node.gswcah

Ortega, E., Giannotta, F., Latina, D., \& Ciairano, S. (2012). Cultural adaptation of the Strengthening Families Program 10-14 to italian families. Child and Youth Care Forum, 41(2), 197-212. doi.org/10.1007/s10566-011-9170-6

Pérez, G. F., \& Lugo, M. (2019). Contexto social que delimita las experiencias reproductivas en la adolescencia. México. http://www.trabajosocial.unam.mx/enfadea/

Pérez, G. F., Lugo, M., \& Manjarres-Posada, N. I. (2019). Uso de métodos anticonceptivos en la adolescencia. Ciudad de México. http://www.trabajosocial.unam.mx/enfadea/

Prochaska, J. O., \& Prochaska, J. (2019). Transtheoretical Model. En J. M. Rippe (Ed.), Lifestyle Medicine (3era ed.). Boca Raton, Florida: Taylor \& Francis.

Rolleri, L. A., Fuller, T. R., Firpo-Triplett, R., Lesesne, C. A., Moore, C., \& Leeks, K. D. (2014). Adaptation guidance for evidence-based teen pregnancy and STI/HIV prevention curricula: From development to practice. American Journal of Sexuality Education, 9(2), 135-154. doi.org/10.1080/15546128.2014.900467

Salinas, S., Castro, M. D., \& Fernández, C. (2014). Vivencias y relatos sobre el embarazo en adolescentes. Panamá. https://www.unicef.org/lac/informes/vivencias-y-relatos-sobre$\underline{\text { el-embarazo-en-adolescentes }}$

Whitaker, A. K., Quinn, M. T., Munroe, E., Martins, S. L., Mistretta, S. Q., Gilliam, M. L., \& (2016). A Motivational Interviewing-based counseling intervention to increase postabortion uptake of contraception: A pilot randomized controlled trial. Patient Education and Counseling, 99(10), 1663-1669. doi.org/10.1016/i.pec.2016.05.011 